\title{
Near Earth Object impact simulation tool for supporting the NEO mitigation decision making process
}

\author{
Nick J. Bailey, Graham G. Swinerd, \\ Andrew D. Morley and Hugh G. Lewis \\ Department Aerospace Engineering, School ofEngineering Sciences, \\ University of Southampton, Southampton, UK
}

\begin{abstract}
This paper describes the development of a computer simulation tool, NEOSim, capable of modelling small NEO impacts and their effect on the global population. The development of the tool draws upon existing models for the atmospheric passage and impact processes. Simulation of the land and ocean impact effects, combined with a population density model, leads to casualty estimation at both a regional and global level. Casualty predictions are based upon the intensity of each impact effect on the local population density, with consideration given to the population inside or outside local infrastructure. Two case studies are presented. The first evaluates the potential threat to the UK, and highlights coastal locations as being at greatest risk. Locations around Cornwall demonstrate an increase in casualties above the local average. The second case study concerns the potential impact of asteroid (99942) Apophis in 2036. Propagation of the possible orbits along the line of variance leads to an extensive path of risk on the Earth. Deflection of the asteroid, by a variety of means, will move the projected impact site along this path. Results generated by NEOSim for the path indicate that South American countries such as Colombia and Venezuela are at a greatest risk with estimated casualty figures in excess of 10 million. Applications of this software to the NEO threat are discussed, along with the next stage of NEO impact simulation development.
\end{abstract}

Keywords. Impact risk

\section{Introduction}

Near Earth Objects have been the subject of continued study for many years with NASA's Spaceguard Survey providing the most comprehensive search and activity to date. Objects greater than $1 \mathrm{~km}$ in diameter are the focus of these surveys, while small sub-kilometre bodies remain largely uncatalogued. This NEO natural hazard was brought to public attention by the recent discovery of (99942) Apophis, a relatively small $320 \mathrm{~m}$ diameter asteroid, as it passed by Earth in December 2004. Following this discovery, the Earth witnessed the largest natural disaster on record when a 9.0 earthquake off the coast of Sumatra generated a tsunami wave which inundated coastlines around the Indian Ocean and parts of Africa. The calculated death toll for this event is 229,866 (United Nations 2006 and was a relatively 'small' tsunami compared to ocean impact generated tsunamis. Both these events demonstrate the significant threat posed by the sub-kilometre NEO impact hazard.

The work outlined in this paper began in the middle of 2004 and has focused on developing a computer simulation tool capable of modelling both land and ocean impact scenarios for sub-kilometre bodies. The program is called NEOSim and generates a casualty estimate by assessing the interaction of the impact generated effects with human populations. Two case studies have been investigated which deal with the local and global 
threat. The first scenario looks at the threat posed to a small region by objects landing in the vicinity. The UK was chosen as the test case, but this methodology can be applied to any location on the globe to assess the local risk. The second scenario investigates potential impact events of the asteroid Apophis along the predicted line of risk for the potential Earth encounter in 2036. This line stretches from Kazakhstan, across the north Pacific and Central America to the Cape Verde Islands in the Atlantic. Analysis shows the potential consequences for a space mission that seeks to mitigate the impact threat by altering the object's orbit.

\section{NEOSim Methodology}

A study of the literature revealed that there was a deficiency in tools that enabled the study of NEO impacts, including both land and ocean impacts. Three distinct phases of an impact were identified to be incorporated into the NEOSim program: atmospheric entry; impact energy transmission; and casualty prediction. These are briefly discussed here.

\subsection{Atmospheric entry}

NEOs impact the Earth at hypersonic velocities (typically in excess of $12 \mathrm{~km} / \mathrm{s}$ ) and, during atmospheric entry, generate complex hypersonic flows around the object. The dominant feature is the high stagnation pressure at the leading edge of the object and the expansion of the bow shock around the body. Behind the shock front the high pressure generates high temperatures and these combined effects lead to a mass loss through ablation of the surface material. If the stagnation pressure difference exceeds the internal strength of the object, which is determined by the physical composition of the NEO, the object will rupture leading to fragmentation. NEOSim incorporates three models for studying the effect of fragmentation.

- Single object model for robust, high strength objects

- Catastrophic fragmentation for weak objects

- Progressive fragmentation for pre-fractured objects

The single object model assumes the NEO remains intact throughout the atmospheric passage with mass loss only through ablation while the catastrophic fragmentation model, developed by Chyba et al. (1993) and Lyne et al. (1996) commonly known as the 'Pancake' model, deals with very low strength objects that disintegrate in the atmosphere. This model is thought to best represent the Tunguska event of 1908. the progressive fragmentation model was developed by Borovička et al. (1998), Baldwin \& Sheaffer (1971) and Foschini (1998) to model objects that are pre-fragmented in orbit with relatively high internal strength. These objects break apart in the atmosphere but don't entirely disintegrate resulting in multiple ground impacts. A pre-defined break-up altitude is implemented according to Klinkrad et al. (2004), set at $30 \mathrm{~km}$.

\subsection{Surface Impact Effects}

During impact the object's kinetic energy excavates a crater before being transmitted through a number of mechanisms identified by Collins et al. (2004). For land impact these mechanism include ejecta distribution, a seismic shock wave, a surface blast wave and thermal radiation generated by an expanding fireball. These impact generated effects can be likened to those associated with nuclear detonations. However, little data from nuclear testing is publicly available. Instead, the models by Glasstone \& Dolan. (1977) and Collins et al. (2005) provide a good approximation and have been implemented in the NEOSim software. 
Ocean impacts account for approximately two thirds of all NEO impact events due to the proportion of Earth covered by water. Ocean impacts are characterised by the excavation of a transient cavity (or crater) through the deposition of the object's kinetic energy at the impact site. This cavity is naturally unstable and immediately in-fills from the surrounding ocean. This in-filling water oscillates vertically generating the tsunami wave train. NEOSim implements the models by Chesley \& Ward (2003) to calculate the tsunami's shoaling characteristics (wave run-up and run-in distances) on surrounding shorelines.

A special case exists where the transient cavity depth is greater than the ocean depth. In this 'bottoming out' scenario both a tsunami and some land impact generated effects are assumed to affect the surrounding region.

\subsection{Casualty Prediction}

Human population density data was obtained from NASA Visible Earth $\dagger$. Although the data set is global, the low resolution limits the accuracy of the casualty estimations. This data is utilised by a dedicated casualty prediction algorithm that has been developed to assess each impact scenario. Following the simulation of an impact, the population in the area affected is used to calculate the casualty figure. The number of casualties at each point is depended on the local population and the severity of each effect. Increasing the distance from the impact site is the main factor in reducing the severity of each land impact effect. Several casualty prediction variables can be manipulated by the user for fine tuning of the scenario, including how many casualties are generated by the increasing effect intensity. Consideration is given to the percentage of people inside or outside local infrastructure, as this will offer some protection, assuming the buildings are not destroyed. The sum of casualties generated by each effect is output as a casualty distribution map.

For ocean impacts, casualty prediction is implemented in a similar manner, with casualties generated along the inundated littoral. The effectiveness of the wave in producing casualties is based upon the run-up height of the wave at shoaling and the run-in distance. The longer travel time of a tsunami provides populations with more time to evacuate, provided that there is a warning system. This casualty-reducing factor is incorporated, based on the wave travel time.

\subsection{Data Output}

NEOSim's primary output is the total casualty figure, which is provided to the user via a pop-up window. The user is then presented with the array of outputs provided by NEOSim. These outputs overlay data concerning each impact generated effect (both for land and ocean impacts) onto a world map. Furthermore, the casualty data is provided as an overlay onto the casualty density map with shading from dark to bright to denote low to high casualty densities. Figure 1 provides an example of the NEOSim outputs with a test impact into the North Sea (denoted by the small cross). The figure is a composition, with the left side showing the impact effects, in this example a tsunami, and on the right side the casualty density map. Verification of the absolute casualty figure for any impact is difficult due to the lack of first hand impact experience and the number of factors involved in casualty generation. Thus, NEOSim focuses on the relative casualty density over the area affected to provide information on the locations at greatest risk and to determine which populations should be evacuated in the event of an impact.

$\dagger$ NASA Visible Earth, Population Density Map, [Online] http://visibleearth.nasa.gov/ view_rec.php? $=116$ [15 March 2005]. 


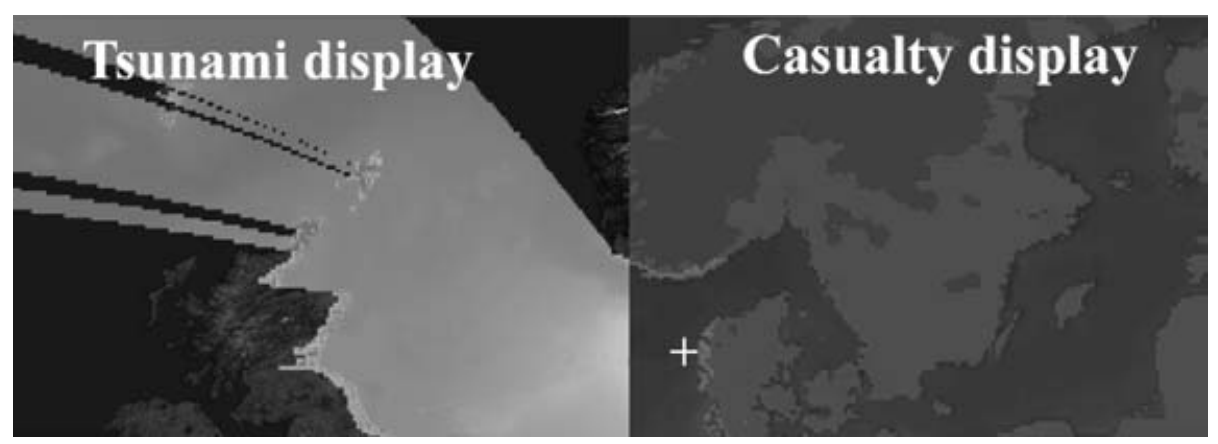

Figure 1. Figure shows the output provided by NEOSim. The left side shows the impact generated effects distribution, here the tsunami wave. The right provides the casualty density map with light regions representing more casualties.

Table 1. Characteristics of the test NEO used in the Case Study One simulation.

\begin{tabular}{lc}
\hline Parameter & Initial Value \\
\hline Radius & $150 \mathrm{~m}$ \\
Impactor Velocity & $25 \mathrm{~km} / \mathrm{s}$ \\
Altitude & $106 \mathrm{~km}$ \\
Density $2600 \mathrm{~kg} / \mathrm{m}^{3}$ & \\
Yield Strength & $10000000 \mathrm{~N} / \mathrm{m}^{2}$ \\
Heat of Ablation & $8000000 \mathrm{~J} / \mathrm{kg}$ \\
\hline
\end{tabular}

\section{Case Study 1 - United Kingdom NEO Impact Risk}

\subsection{Methodology}

In order to study the effect of NEO impacts around a region on the Earth rather than simply a single impact, a multi-run application was built into NEOSim. This multi-run tool allows the user to select a region defined by a latitude and longitude bin over a particular location of interest. This region is then divided into a number of cells (defined by the user) into which a single object is impacted. The casualty figure output from each cell impact is used to shade the output map. Mapping the results in this manner avoids the problem of validation of any specific casualty figure by providing the relative risk of an impact into each cell. For this case study the UK was chosen as the target with the latitude bin from $40^{\circ}$ to $70^{\circ}$ north and longitude bin from $30^{\circ}$ west to $15^{\circ}$ east. Resolution of the grid cells (the number of cells that fit within in the latitude longitude bins) is restricted by the processing time required for the simulation. Increasing the resolution and therefore number of cells improves the quality of the data but requires increasingly long runtime. For the present case study a 400 cell grid was chosen as a compromise between runtime and quality. The characteristics of the incident NEO are defined in Table 1.

\subsection{Results}

Figure 2 presents the results for the UK case study. The results show that the UK population is at greatest risk from objects impacting in the Atlantic Ocean to the west and south west. The peak amplitude of the tsunami is dependent on the depth of water at the point of impact with deep transient craters resulting in large amplitude oscillation as the cavity in-fills. Impacts into shallow oceans bottom out preventing the large oscillations of water, generating only small tsunami waves. This explains the low expected casualties 


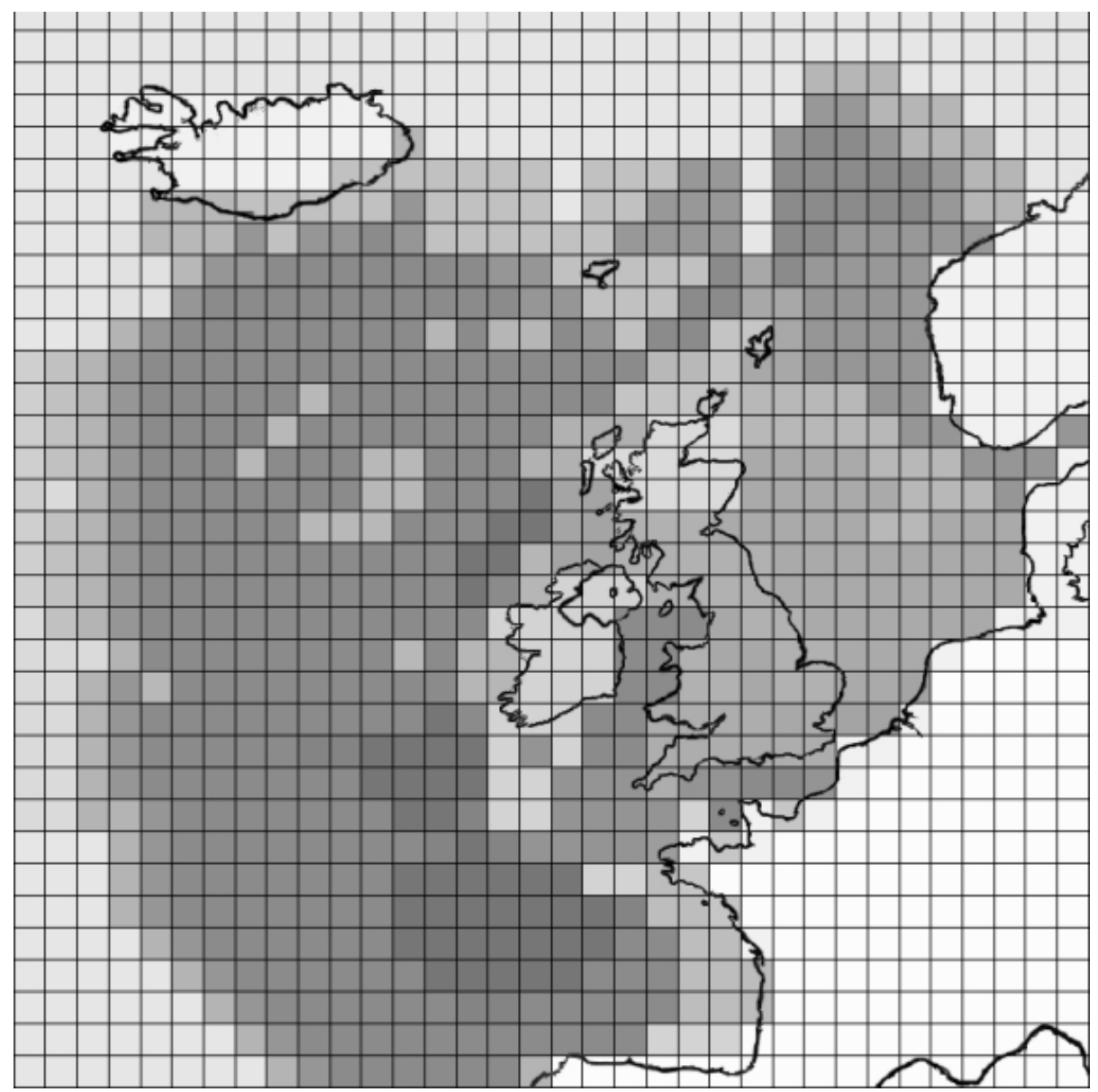

Figure 2. Shading denotes the number of casualties by the impact in the centre of each cell with dark shading representing more casualties.

from impacts into the North Sea where the water is only $30 \mathrm{~m}$ deep at maximum removing the potential to generate large waves. This provides some protection to communities on the eastern seaboard against tsunami inundation.

Populations highlighted as being at greatest risk are those along the westward coastline of Ireland and the south-western regions of the UK. In particular Cornwall and Devon, with a historically high dependence on maritime fishing industries, are at very high risk from such deep ocean impacts. NEOSim's tsunami model does not include wave diffraction which will reduce the effectiveness of the tsunami's propagation along the English Channel. Furthermore, the Bristol Channel, which experiences the second greatest tidal range in the world along with a large bore phenomenon, is likely to amplify the tsunami's amplitude as it travels up the constricting passage. Thus low-lying regions along the Severn Estuary are likely to experience catastrophic inundation from any deep Atlantic Ocean impact to the south west. Central coastlines of England and Wales, and in particular the major port of Liverpool, are shown at a reduced risk. This is explained by the protection afforded by Ireland which receives the majority of the inundation from impact tsunamis. 


\subsection{Conclusions}

The threat to the UK from small, Earth-impacting asteroids is predominantly from Atlantic Ocean impacts to the west and south west. Atlantic impacts to the south west of the UK represent the greatest hazard with populations along the south west coastlines of the UK, including Cornwall, Devon and south Wales at greatest risk. A number of major cities are situated along the Bristol Channel including Cardiff, Newport, Bristol and Gloucester. These large cities will be at an increased risk due to the funnelling effect of the channel. It is expected that communities along the South coast of England will also be at increased risk due to the funnelling of the tsunami waves as they move up the Channel. Impacts in the shallow waters of the North Sea and Irish Sea present a much reduced casualty potential due to the small tsunami generated. These regions help to lower the risk to the UK by increasing the Earth's area where impacts can occur with little consequences. Land impacts in the UK will be very rare due to the small total land area (approximately $0.1 \%$ of the Earth's surface area). The consequences, while potentially severe even for these small NEOs, tend to be localised. The typically high population density of England in particular increases the risk of many casualties resulting from a land impact. NEOSim estimates casualty figures for England land impacts to be from 3 to 8 million, which is comparable to London's population of 7.5 milliont. The cell that was created over London actually shows a larger casualty signal.

While this study focuses solely on the threat to the UK, in reality it is impossible to ignore the shared threat to other countries in the region. Particularly important in this study is the consequence for Ireland. When considering simply the UK, Ireland acts as a barrier to block the majority of tsunami waves from reaching the shores of England and Wales, effectively lowering the risk. However, it would be impossible not to view such impacts as threatening when, living in as we do in the global community, the consequences for neighbouring countries would be significant.

The high threat to the UK from Atlantic Ocean impacts calls for increased research into mitigating the threat. Furthermore, considering the very small risk from tectonic and volcanic activity and even catastrophic weather (such as hurricanes), the NEO risk is significant, potentially the UK's single greatest natural hazard (apart from, perhaps, the long-term effects of global warming).

\section{Case study 2-Apophis Path of Risk}

The probability of impact for the asteroid 99942 Apophis has been reduced to 1 in 30,000 (equivalent probability of $3.3 e^{-5}$ ) and thus no longer presents a significant risk. However, the example of Apophis has been used to demonstrate the potential of studying an object's path of impact risk. The speed of the simulation enables studies of newly discovered risk paths to be performed quickly to provide an assessment of the potential consequences of the impact.

\subsection{Methodology}

In order to study the effect of the potential impact of Apophis, the line of risk was required in latitude/longitude coordinates. Work by the B612 Foundation [footnote 1: website URL] has generated a path of risk for the predicted impact in 2036 using orbit intersections. The developed path is shown in Figure 3, which was processed to generate the latitude and longitude coordinates for every point along the path. A fixed longitude division was used to produce equal cell widths. NEOSim's multi-run tool was extended

$\dagger$ Wikipeda London [Online] http://en.wikipedia.org/wiki/London [15 August 2006]. 


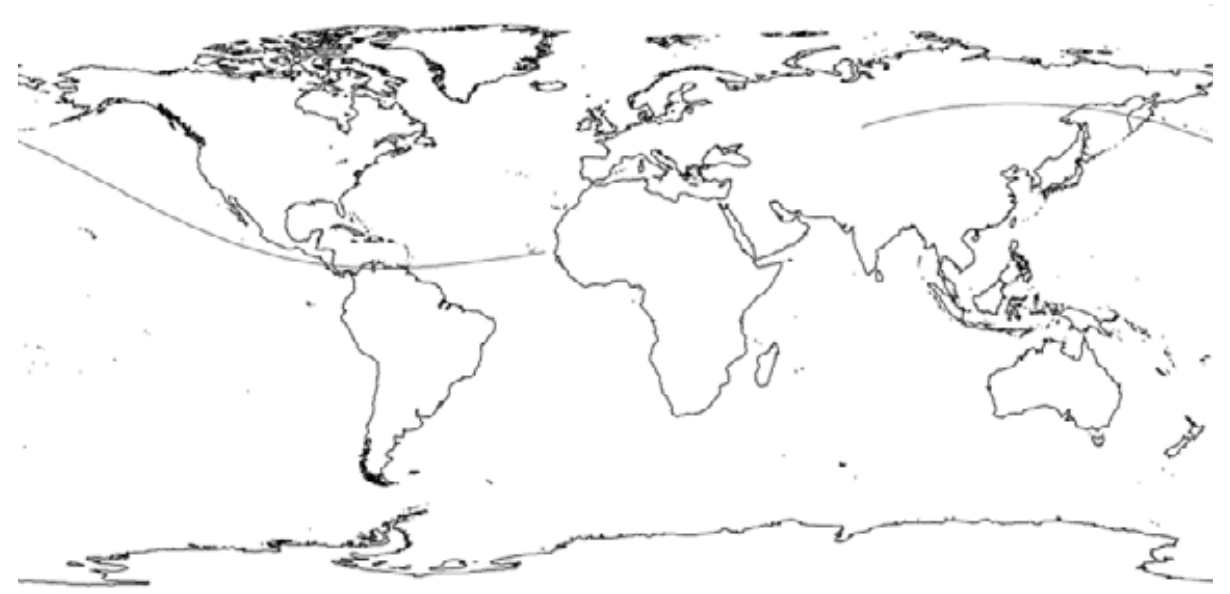

Figure 3. The curved line is the calculated path of risk for the potential impact of asteroid 99942 Apophis in 2036. This line is determined using orbit integrations.

Table 2. Assumed physical characteristics of the asteroid Apophis used as the test impactor. Data from http://neo.jpl.nasa.gov/risk/a99942.html.

\begin{tabular}{lc}
\hline Parameter & Initial value \\
\hline Radius & $320 \mathrm{~m}$ \\
Impactor Velocity & $12.65 \mathrm{~km} / \mathrm{s}$ \\
Object Density & $2600 \mathrm{~kg} / \mathrm{m}^{3}$ \\
Type & Monolithic \\
\hline
\end{tabular}

to model a series of impact cells along the path. A single 1-sigma cell was added above and below the path to represent the uncertainty in the lateral direction.

The estimated characteristics of Apophis, given in Table 2, were used to define the impacting object in NEOSim. This object was impacted into the centre of each cell along the path. The casualty figure for each impact was used to shade each cell as in the first case study.

\subsection{Results}

The western and eastern limbs of the modeled risk path are shown in Figures 4 and 5 respectively. For publishing reasons the shading has been converted to a grey scale with black representing most casualties and white least. The western limb of the path stretches from the Pacific Ocean, through Kamchatka and into Central Russia. The region of greatest casualty generation is found at the path's most westerly point with impacts into Kazakhstan. The second most significant region of risk is associated with impacts into the Sea of Okhotsk, north of Japan. Here impacts generate a tsunami that inundates the populated coastal region of Hokkaido, Japan, generating many casualties. Land impacts over northern parts of Russia appear to produce relatively few casualties.

The eastern limb of the path show ocean impacts into the Pacific far from land generating relatively few casualties compared to those close to populated land masses such around Central America mainland or the Hawaiian archipelago. Impacts around the coast of Central America (up to a range of 1200 kilometres, [750 miles]) and in the Gulf of Mexico represent the greatest threat to human life, indicated by that dark shading in 


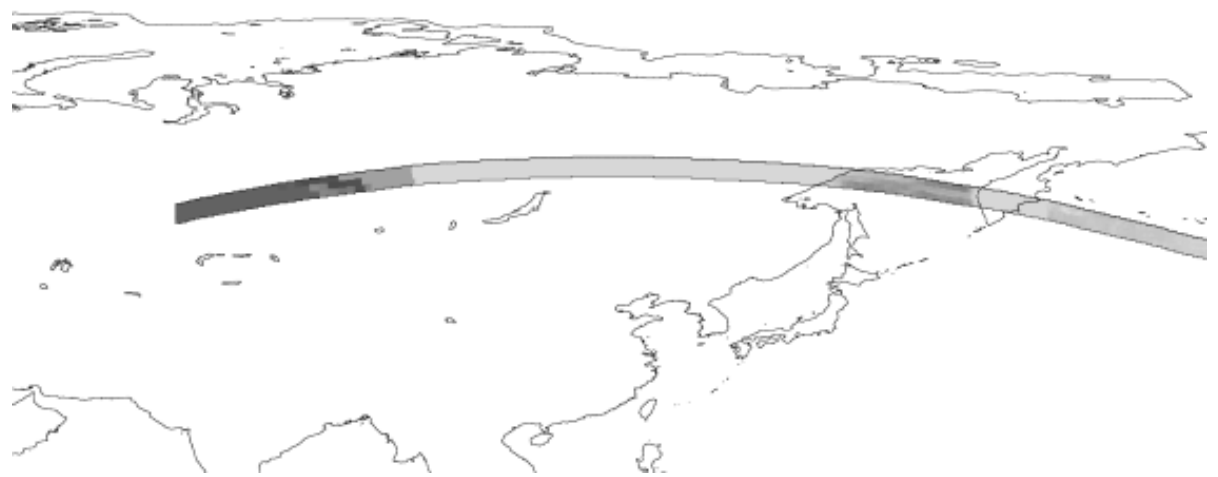

Figure 4. This figure presents the western limb results of the Apophis risk path. The path stretches from Kazakhstan, through northern Russia and into the Pacific Ocean. Cells shaded dark represent higher casualty figures generated from the impact into the centre of the cell.

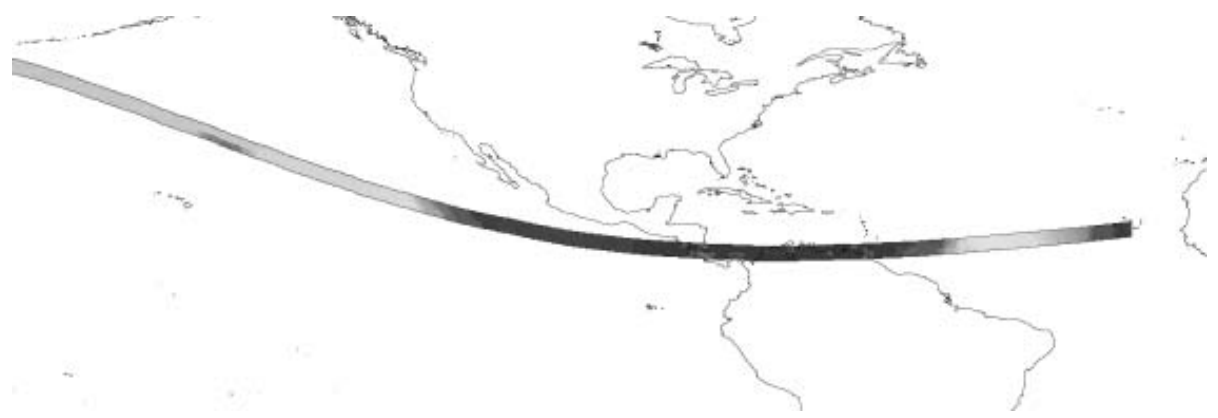

Figure 5. This figure presents the eastern limb of the Apophis path of risk stretching from the Pacific Ocean across Central America to the Cape Verde Islands in the Atlantic Ocean. Dark shaded cells are those which generated most casualties from the impact into the centre of that cell.

Figure 5. These casualties are generated along the populated coastal regions of Central America.

Land impacts in this region across Central America show some interesting features. In general these impacts generate fewer casualties than the surrounding ocean impacts. However, particular impacts in Venezuela produce casualty estimates comparable to these oceanic events. This variability is due to the location dependence of land impacts. For example a direct impact into Caracas will produce many more casualties than an impact only a few kilometres outside the city. Overall the land impacts in Columbia and Venezuela generate more casualties than those in the Central American countries of Nicaragua, Costa Rica and Panama.

The most easterly potential impact sites approach the Cape Verde Islands off the western coast of Africa. Impacts at this end of the path generate relatively high casualty figures. The generated tsunami affects the islands themselves as well as coastal communities of Mauritania, Senegal and Gambia.

\subsection{Conclusions}

NEOSim demonstrates that, at both ends of the risk path, there exists a region. Deflection of the asteroid impact site in either direction will increase the threat to either one of these two regions. Thus an assessment would need to be made to determine which of these populations would most feasibly accept the increasing risk. In either case the mitigation 
mission launched needs to be fully capable of deflecting the asteroid's orbit to prevent the object 'just hitting' and thus catastrophically affecting either of these two populations.

Of interest are the relatively low casualty estimates for Pacific and Atlantic Ocean impacts far out to sea. These impacts, while appearing to have little consequence, still generate many casualties and are a significant hazard. However, relative to impacts around Central America, these ocean events represent a significantly lower threat. Impacts close to Hawaii are highlighted as generating more casualties. Warning time is a factor that will dramatically reduce the number of casualties resulting from an ocean impact. Accurate prior knowledge of the impact site combined with the long travel time of the tsunami wave (of the order of hours) will aid in evacuation and reduce the total casualty figure. This will help prevent scenes such as those witnessed during the Sumatran earthquake induced tsunami of 2004 .

\section{The Application of the NEOSim tool to aid the Decision Making Process}

\subsection{The UK Risk Case Study}

Studying a specific region on the Earth allows for an assessment of what form the risk to the region or a particular country in that region takes. The NEOSim output indicates whether the country's population is at greater risk from ocean or land impacts and which impacts will generate most casualties. In the case of the UK, the threat is greatest from ocean impacts and the generated tsunami waves. Many factors are involved in determining this threat, particularly the amount of coastline exposed to deep ocean, the number of coastal populations and the local topography and bathymetry.

The UK is protected from many possible Atlantic Ocean impacts by the presence of Ireland. However, it is impossible to segregate the study to one particular country as, while the majority of Wales is protected by the presence of Ireland, the western communities of Ireland will be severely affected. Therefore it is impossible to study the consequences for a whole region as many countries will be affected by any impact.

The threat mitigation decision making process for the UK concerns populations are protected from the NEO impact hazard. NEOSim demonstrates that the greatest threat is faced by tsunami inundation from Atlantic Ocean impacts. Thus, to mitigate this threat, research is required into methods for protecting the major cities (indicated previously) from this inundation. One feasible method would be through a tsunami warning system with evacuation strategy put in place for each major city. Thus, in the event of an impact, the most densely populated regions at risk places could be evacuated to reduce the overall loss of life.

\subsection{The Apophis Risk Case Study}

Despite the recent orbit refinement of the asteroid Apophis essentially removing its impact threat for 2036, the experience highlights the potential for Earth to be hit unexpectedly by a small NEO. Thus there is a real need for rapid assessment of the impact consequences and determination of the populations at greatest risk. Examining the Apophis path of risk demonstrates the dramatic consequences for an impact. The study highlights regions around Central America as being at greatest risk from the asteroid. Therefore any mitigation attempt would most likely focus at moving the asteroid's orbit away from this region. However, any mitigation manoeuvre will always have the effect of increasing the risk to one region over another. In this example, large populations at risk are highlighted at each end of the path. Thus any mitigation mission will move the asteroid towards one of these regions. 
Decisions regarding the relative importance of one community over another are difficult to make, but need to be made before any mitigation scenario is attempted in terms of modifications of a NEO's orbit, with resultant change in impact site. Such discussion would be required at an international level as the consequences are truly multi-national. Application of the NEOSim tool enables easy assessment of various scenarios quickly and cheaply to inform this discussion as well as highlighting which countries will be affected.

\subsection{Future Work}

Work has been ongoing to develop an advanced impact simulator called NEOimpactor which improves on NEOSim in three major areas:

- The inclusion of an infrastructure damage model to determine the economic cost of an impact event,

- The advancement of the tsunami model from a ray tracing method to a neural network approach to cater for diffraction around coastlines, and

- The development of a database software architecture to enable manipulation of data layers to power new and novel investigations. One application of this database model is to provide feedback about the effects suffered by only one pre-selected country.

Early results have been compared to the outputs of NEOSim for equivalent impact events and also compared to real world scenarios including the Sumatra Indian Ocean tsunami.

\section{Acknowledgements}

We would like to thank Professor R. Crowther, the principal sponsor of the project, for his useful input and advice through out.

\section{References}

United Nations Office of the Special Envoy for Tsunami Recovery, [Online] available from: http://www.tsunamispecialenvoy.org/country/humantoll.asp [19 July 2006]

Chyba, C.F., Thomas, P.J. \& Zahnle, K.J. 1993, Nature 361, 40

Lyne, J.E., Tauber, M. \& Fought, R. 1996, J. Geophys. Res. 101, 23207

Borovička, J., Popova, O.P., Nemtchinov, I.V., Spurný, P. \& Ceplecha, Z. 1998, Astron. Astrophys. 334, 713

Baldwin, B. \& Sheaffer, Y. 1971, J. Geophys. Res. 76, 4653

Foschini, L. 1998, Astron. Astrophys. 337, 5

Klinkrad, H., Fritsche, B. \& Lips, T. 2004, International Astronautical Congress 2004, vol. IAC04-IAA.5.12.2.07

Collins, G.S., Melosh, H.J. \& Marcus, R.A. 2004, Lunar and Planetary Laboratory, [Online] www.lpl.arizona.edu/ImpactEffects [28 January 2005]

Glasstone S. \& Dolan P.J. 1977, The Effects of Nuclear Weapons, 3rd Edition, United States Department of Defence

Collins, G.S., Melosh, H.J. \& Marcus, R.A. 2005, Meteoritics \& Planetary Science 40, 817

Chesley, S.R. \& Ward, S.N. 2003, AAS/Division for Planetary Sciences Meeting Abstracts, vol. 35

Ward, S.N. \& Asphaug, E. 2000, Icarus 145, 64

National Aeronautics and Space Administration 2003, [Online] http://visibleearth.nasa.gov/ view_rec.php?id=116 [15 March 2005] 